\title{
Management of the brain-dead organ donor
}

\author{
Caitlin Clarke ${ }^{1}$
}

Received: 8 March 2021 / Revised: 13 May 2021 / Accepted: 3 June 2021 / Published online: 17 September 2021

(c) Indian Association of Cardiovascular-Thoracic Surgeons 2021

\begin{abstract}
Organ transplantation is a life-saving intervention for patients suffering from end-stage organ failure, but it relies on the availability of donor organs. However, even when donors are available, the brain-dead organ donor is a clinically complex patient who presents many management challenges. Donor management with a goal of optimization of organ function is essential to maximizing the number of patients who can be helped by each individual donor. Thoughtful critical care management of the potential organ donor, with a focus on meeting donor management goals, can lead to improved donation outcomes.
\end{abstract}

Keywords Organ donation $\cdot$ Donor management $\cdot$ Critical care $\cdot$ Brain death

\section{Introduction}

Currently one of the biggest challenges in providing the opportunity for a life-saving organ transplant to a patient in need is that the number of patients waiting for a life-saving transplant far outweighs the number of available organs. Their survival depends on the availability of donor organs, but there remains a huge disparity between the number of available organs and the number of waiting recipients. For example, in the USA, per the Organ Procurement and Transplantation Network (OPTN), as of January 2021, there are over 108,000 people waiting for a life-saving organ transplant. Even with the number of deceased donors increasing year to year, with a record number reached in 2020 at over 12,000 donors, the numbers are still so far from having enough donors to help everyone waiting. Because of this, it is absolutely vital that every donor be meticulously managed so that they can save as many recipients' lives as possible, but the management of the organ donor can present many challenges. These are clinically complex patients, and management goals are centered on optimizing organ function while working to balance the optimal homeostasis preferred for each individual organ and dealing with the physiological effects of brain death.

Gift of Life Donor Program (GLDP) has led the USA with the highest number of donors for the past 13 consecutive

Caitlin Clarke

cclarke@donors1.org years. In addition, GLDP's annual donation rate of 55 organ donors-per-million-population is among the highest in the world [1]. We have obtained this success through collaboration with our healthcare team partners and meticulous management of potential organ donors. While we are proud of our progress, we continue to strive for improvement, optimizing each organ donor's potential to provide quality organs for transplant using the management strategies reviewed below.

The potential brain-dead organ donor is a patient in the intensive care unit (ICU) who has suffered a catastrophic brain injury. Some examples being a patient with an anoxic brain injury, head trauma, or a cerebral vascular accident, among others. Prior to the pronouncement of brain death, this patient is being managed by the intensivist and the ICU team. During their time in the ICU, there will be a point when it is deemed that this care is futile in terms of saving the life of this patient. At this point it is still crucial that the healthcare team avoid a deceleration in maintaining hemodynamic stability. While they may not be able to save the life of that patient, it is imperative that the care team take into consideration the lives that could be saved by that individual, if they are able to go on to organ donation. Continued aggressive care by the ICU team, not only preserves the opportunity for organ donation for the patient and their family, but can significantly impact the organ function and the ultimate organ yield, if that patient goes on to be a donor. Through the management of this patient, the ICU team plays an active role in providing the gift of life to others [2].

1 Gift of Life Donor Program, Philadelphia, PA, USA 
Once death by neurological criteria has been determined by the medical team caring for the potential organ donor, and the authorization for organ donation has been obtained from the donor's next-of-kin, the organ procurement organization (OPO) will collaborate with the intensivist and the ICU medical team on the clinical management of the donor. At this time the focus of the clinical care becomes to evaluate and optimize organ function to result in the maximum number of recipient's lives saved.

Even patients with complex medical histories have the potential to save lives. A thorough evaluation of the potential donor's medical and social history as well as their current hospital course and clinical status is critical for determining the appropriate management interventions for each donor. This will also ensure providing comprehensive information to the transplant surgeon, who will be evaluating the suitability and safety of the organs for transplant.

\section{Complications of brain death}

Brain death is defined as the irreversible loss of all functions of the brain, including the brainstem. The three essential findings in brain death are coma, absence of brainstem reflexes, and apnea. An evaluation for brain death should be considered in patients who have suffered a massive, irreversible brain injury of identifiable cause. A patient determined to be brain dead is legally and clinically dead [3].

As a patient with a devastating neurological injury progresses to brain death, there is a high risk for hemodynamic instability. With impending brain stem herniation, there is a massive release of catecholamines as the body responds to the increase in intracranial pressure and attempts to preserve brain perfusion by increasing cerebral perfusion pressure, thus, resulting in autonomic dysfunction, vasoconstriction, and diminished end-organ perfusion. This autonomic storm will also cause neurogenic pulmonary edema and trigger a systemic inflammatory response in the patient. Subsequently, cerebral herniation leads to the loss of the sympathetic nervous system, causing marked vasodilation with a fall in cardiac output, hemodynamic instability, hypotension, hormonal failure, and metabolic acidosis. As neuronal cell death progresses, this leads to endocrine failure, causing diabetes insipidus and diuresis, which results in hypernatremia and hypovolemia. There is also a decrease in thyroid-stimulating hormone (TSH) secretion which allows for impaired cardiac contractility and shifts in metabolism, resulting in increased lactate and acidosis. Additionally, donor stress responses are lessened contributing to further hypotension and cardiovascular instability. In summary, the most prevalent systemic complications post brain death include cardiac arrhythmias, diabetes insipidus, disseminated intravascular coagulation, hypotension, neurogenic pulmonary edema, systolic myocardial dysfunction, and thrombocytopenia, among others. All of these factors can threaten organ viability, and therefore much of the organ donor management, post brain death, focuses on the normalization of these derangements [4-6].

\section{Hemodynamic management}

Overall goals in the management of the brain-dead donor include maintaining euvolemia and optimal perfusion pressure with a mean arterial pressure (MAP) of at least $60 \mathrm{mmHg}$, urine output of at least $1 \mathrm{ml} / \mathrm{kg} / \mathrm{hr}$, and left ventricular ejection fraction (LVEF) of at least $45 \%$. See Table 1 for details [2].

Hypovolemia is frequently present at brain death and should be addressed prior to pursuing other interventions. Recommendations for volume replacement include isotonic crystalloids, as well as $5 \%$ albumin, for more rapid volume expansion, if necessary [2, 7]. Hydroxyethyl starch is not recommended for use in organ donors as it has been shown to be associated with delayed graft function in kidney recipients [8]. Packed red blood cells should be given in the presence of anemia that may diminish the blood's ability to provide adequate oxygenation to vital organs. Other blood products, such as fresh frozen plasma, platelets, cryoprecipitate, can be used to manage bleeding. Blood product administration also has the benefit of providing additional volume replacement [2].

If, after adequate volume resuscitation hypotension, as evidenced by a $\mathrm{MAP}<60 \mathrm{~mm}$ of $\mathrm{Hg}$, persists, then vasopressor support is necessary to maintain adequate organ perfusion. Low-dose dopamine has frequently been preferred as the first-choice agent due to its inotropic and vasopressor effects. There have been additional benefits seen in transplant outcomes after use of dopamine supporting its preferential selection. It has been found to protect organs against ischemia/reperfusion injury and inflammation by stimulating the induction of enzymes such as heme oxygenase-1 [9]. In 2009 Schnuelle et al. [10] found that using low-dose dopamine reduced the need for dialysis after kidney transplantation. Additionally, in 2011 Benck et al. [11] deduced that the

Table 1 Donor management goals

\begin{tabular}{ll}
\hline Heart rate & $60-120 \mathrm{bpm}$ \\
Mean arterial pressure (MAP) & $>60 \mathrm{mmHg}$ \\
Urine output & $1-3 \mathrm{ml} / \mathrm{kg} / \mathrm{hr}$ \\
LV ejection fraction & $>45 \%$ \\
P/F ratio for lung donors & $>300 \mathrm{mmHg}$ on PEEP 5 \\
Central venous pressure (CVP) & $6-8 \mathrm{mmHg}$ \\
Temperature & $36-38$ degrees \\
\hline
\end{tabular}


treatment of brain-dead donors with low-dose dopamine (4 $\mathrm{mcg} / \mathrm{kg} / \mathrm{min}$ ) does not harm cardiac allografts, but appears to improve the clinical course of the heart recipient. Vasopressin also has benefits as a preferential pressor choice, because it not only provides systemic vasoconstriction and improves vasodilatory shock, but can also be used as a treatment for diabetes insipidus (DI), both of which are frequently seen post brain death. In cases of severe shock when additional support is needed, phenylephrine or norepinephrine is recommended for vasodilatory shock components, and epinephrine or dobutamine may be used for cardiac pump dysfunction [2].

Additional hemodynamic monitoring can be beneficial in aiding assessment of volume status and response to therapy. This can be done via central venous catheter, pulmonary artery catheter, or arterial line and provide continuous measurements of central venous pressure (CVP), pulmonary artery occlusion pressure (PAOP), stroke volume, or cardiac output and index (CO/CI) [2].

\section{Hormone replacement therapy and steroid management}

Due to the resulting hormonal failure after brain death, hormone replacement therapy (HRT) with thyroid hormone, steroids, and vasopressin is widely used to counteract the potential detrimental effects of hormone loss associated with brain death and is generally advocated. However, this has not always been entirely supported and does remain controversial [6].

In 2014 Novitzky et al. [12] published a retrospective analysis of brain-dead organ donors over a 10-year period. Based on this review, they felt the data strongly supported the use of T3/T4 therapy, particularly if combined with vasopressin and corticosteroids. They stated this should be considered for all brain-dead organ donors. They found a significant increase in the number of procured organs, with no impact, positive or detrimental, post-transplant.

Much of the evidence supporting the use of HRT has come from animal models, but in 2005 Powner and Hernandez published a review of publications that evaluated the administration of thyroid hormone in adult human donors. They concluded that recommendations for donor care may include thyroid hormone administration, but did not find advocation for routine thyroid hormone replacement for all donors. If a thyroid protocol was adopted, it should also include steroid administration. As well, per their review, treatment with vasopressin, while it was not fully reviewed, may be helpful [13].

More commonly agreed upon is the use of HRT in hemodynamically unstable donors with refractory shock, who require high doses of vasoactive medications despite fluid resuscitation, or potential cardiac donors with an LVEF $<45 \%$. This has resulted in improved outcomes with more transplantable organs on such donors $[2,9]$. Administration of high dose corticosteroids has been shown to decrease vasopressor requirements, as well as improve oxygenation by reducing brain death-induced inflammation $[2$, 5].

In cases of thoracic management when over-aggressive fluid management is not recommended, there have also been benefits shown of using HRT with a CVP $<10 \mathrm{~cm}$ of water, with findings supporting that the utilization of the hearts and lungs for transplantation can still be increased, without negatively impacting other organ systems [14].

Dosing recommendations for thyroid replacement include levothyroxine (T4) $20 \mathrm{mcg}$ intravenously (IV) followed by $10 \mathrm{mcg} / \mathrm{hr}$ or triiodothyronine (T3) $4 \mathrm{mcg}$ IV followed by $3 \mathrm{mcg} / \mathrm{hr}$, and for steroid administration hydrocortisone $300 \mathrm{mg}$ IV or methylprednisolone $1000 \mathrm{mg} \mathrm{IV}, 15 \mathrm{mg} / \mathrm{kg}$ $\mathrm{IV}$, or $250 \mathrm{mg}$ IV followed by infusion at $100 \mathrm{mg} / \mathrm{hr}$ [2, 5].

\section{Heart considerations}

As discussed in the complications of brain death, the herniation process induces a catecholamine storm in the potential organ donor. Physiologically, this results in severe hypertension and tachycardia, followed abruptly by vasodilation, decreased cardiac output, and hypotension. This process precipitates a high incidence of cardiac stunning and cardiac dysfunction in brain dead organ donors. Also, the hormone changes that occur after brain death are thought to further impair myocardial contractility. These factors are felt to be the cause the left ventricular (LV) dysfunction frequently seen after brain death [15].

The first step in evaluation of the patient as a potential heart donor begins with obtaining a transthoracic echocardiogram (TTE). If a TTE is unable to be performed, or inconclusive, a transesophageal echocardiogram (TEE) can be done. For accurate evaluation of the donor's current heart function, and because of the effects brain death can have on the heart, this echocardiogram (ECHO) is obtained after brain death has been determined. Ideally management goals of adequate fluid resuscitation and minimal vasopressor support are met prior to the evaluation of heart function. If cardiac dysfunction is noted on the initial ECHO, additional management may be necessary, followed by additional ECHOs to monitor response to interventions. HRT, as discussed, is recommended for its potential to resolve LV dysfunction.

Further testing via cardiac catheterization is recommended if the patient is at a higher risk for cardiovascular disease based on age ( $>40$ years old) or medical/social history [2]. 
A study by Dhar et al. [15] was completed to evaluate the impact of IV thyroxine infusion to improve post brain death myocardial recovery with hopes of improving utilization of hearts for transplant. While the study was small in size, and did have limitations, their findings saw an increase in LVEF in both the donors who received $\mathrm{T} 4$ and those who didn't, suggesting that the myocardial dysfunction seen after brain death is due most significantly to reversible cardiac stunning and less likely attributed to thyroid, with an improvement seen over time post brain death with hemodynamic management. However, a larger study would need to be completed to confirm these findings.

There was also a study completed by Peled et al. [16] that suggested that donor treatment with thyroid hormone therapy was associated with an increased risk of early graft loss in the heart transplant recipient. It was hypothesized that this was due to a thyroid hormone withdrawal effect that could be potentially mitigated with administration of thyroid hormone to the recipient; however further studies would need to be done. The study limitations did include lack of detail about the thyroid administration in the donor prior to recovery, including dosing and time of administration, but ultimately their findings, as they were, do support the withdrawal hypothesis. Additionally, for recipients who survive the early post-transplant phase, there may actually be favorable outcomes associated with thyroid administration.

Most significantly, it has been demonstrated that even an initial low LVEF, post brain death, does not preclude a donor from becoming a cardiac donor, as there is potential for the heart function to improve in time with adequate management.

\section{Lung considerations}

The scarcity of donors, as previously discussed, is perhaps the most dire when considering lung transplantation, because of the low number of donors who go on to donate their lungs. Per OPTN data as of January 18, 2021, only $25 \%$ of all brain-dead donors in the USA in 2020 were lung donors [17]. Because the opportunity for lung donation can be so scarce, it is crucial that every donor be thoroughly vetted for their potential for lung donation to maximize lung procurement rates. These patients must be meticulously managed, if one entertains the hope of increasing the potential lung donor pool.

It has been widely agreed upon that the ideal donor lung selection criteria include, among other factors, a $\mathrm{PaO} 2 / \mathrm{FiO} 2$ (p/f) ratio of $>300 \mathrm{mmHg}$ on $5 \mathrm{~cm} \mathrm{H} 2 \mathrm{O}$ positive end-expiratory pressure (PEEP) $[2,6,18]$. However, this does not immediately rule out any potential donors that do not meet that criteria on initial assessment, it just indicates the need for more aggressive lung management, in an attempt get the lungs functioning at a level, where they would be deemed suitable for transplant. There are multiple factors that can have a negative impact on the $\mathrm{p} / \mathrm{f}$ ratio and that also have the potential to be reversed, including pulmonary edema, atelectasis, and secretions. The potential lung donor should be evaluated for these complications, with a goal of improving oxygenation and ultimately getting the lungs to a point, where they would be acceptable for transplant.

The initiation of intensive donor management protocols have been shown to improve oxygenation and lung utilization [19]. These protocols should not be limited to donors with initially low $\mathrm{p} / \mathrm{f}$ ratios however, as they can maintain good lung compliance throughout other management interventions that may have an otherwise detrimental effect [20]. These include recommendations for ventilatory settings. While traditional practices for ventilation of a brain-dead organ donor included increased tidal volumes (TV) up to $10-15 \mathrm{ml} / \mathrm{kg}$, after studies for acute respiratory distress syndrome (ARDS), which indicated the injurious effect of higher tidal volumes in critical care, there has been some discussion of reassessing the practice in donor management. Mascia et al. [21] investigated a protective lung strategy TV 6-8 $\mathrm{ml}$ and PEEP 8-10 as well as recruitment maneuvers after any disconnection from the ventilator. While this study was not completed, they did show success in increasing donor eligibility and therefore did endorse these settings in their donor management recommendations, an endorsement that has been reiterated in additional publications [21, 22].

Beyond ventilation recommendations, donor management protocols include apnea testing with continuous positive airway pressure (CPAP), to prevent alveolar collapse, patient positioning with raised head of bed, optimal volume status with prevention of fluid overload, steroid administration, and performing recruitment maneuvers to treat atelectasis and after any time the donor is disconnected from the ventilator $[21,23]$. Specific recruitment strategies vary, but include the basis of short periods of elevated PEEP, to correct and prevent further atelectasis. Minambres et al. [20] recommended controlled ventilation (with a peak pressure limit of $35 \mathrm{mmHg}$ ) and PEEP $18-20 \mathrm{~cm} \mathrm{H} 2 \mathrm{O}$ for $1 \mathrm{~min}$, decreased by 2 each minute, followed by increase of tidal volumes by $50 \%$ for 10 breaths. Van Raemdonck et al. [24] endorsed a strategy of pressure control ventilation with an inspiratory pressure of $25 \mathrm{~cm} \mathrm{H} 2 \mathrm{O}$ with PEEP $15 \mathrm{~cm} \mathrm{H} 2 \mathrm{O}$ for $\sim 2 \mathrm{~h}$ before returning to conventional ventilation. Maciel et al. [5] made the recommendation of recruitment with $30 \mathrm{~cm}$ $\mathrm{H} 2 \mathrm{O}$ of PEEP for $30 \mathrm{~s}$. Also of importance is to be cautious of hemodynamic status and optimize blood pressure prior to any maneuver to prevent any significant hypotension [5].

A restrictive fluid balance, and therefore diuresis, is frequently indicated in management of the potential lung donors due to the high incidence of neurogenic pulmonary edema, post brain death. Aiming for a lower CVP 
and fluid balance has been shown to be beneficial for lung transplantation, without being detrimental to other organ systems [14, 23].

In addition to serial arterial blood gases to evaluate $\mathrm{p} / \mathrm{f}$ ratio, a bronchoscopy should be performed on all donors. This will serve not only as a diagnostic assessment of lung anatomy, but also to assess for any potential aspiration or infection and therapeutically clear any secretions, mucous plugs, or clots that may be present and impeding oxygenation. A bronchoalveolar lavage should be utilized to clear the airways. An assessment of any secretions found should be provided with attention paid to the consistency of the secretions, and if they are able to be fully cleared or if re-welling occurs. The collected specimen should then be sent for gram stain evaluation to assess for infection or presence of increased neutrophils [2].

When attempts to improve lung function are not successful, during the management of the donor in the ICU, there may still be potential for transplant, if the lungs are recovered and ex vivo lung perfusion (EVLP) is utilized. EVLP allows for a more complete assessment of lungs that may initially seem unsuitable for transplant and also has the potential for further treatment of these lungs to a point of being clinically acceptable for transplant [25].

\section{Liver, kidney, and pancreas considerations}

Management of the donor includes optimized hemodynamics, fluid resuscitation, normalized electrolytes, and adequate urine output of 1-3 ml $/ \mathrm{kg}$. Frequent assessment of chemistry panels include liver function testing, amylase, lipase, coagulation studies, complete blood counts, urinalysis, as well as arterial blood gases for monitoring of acid base balance and oxygenation. Hemoglobin A1C can aid in evaluation of the pancreas for transplant.

Much of the management for a potential liver donor is aimed at lowering, or normalizing, serum sodium levels. Hypernatremia is frequently seen post brain death for a number of reasons, including diabetes insipidus and hypovolemia, as well as hyperosmolar treatments prior to brain death. This has been demonstrated to be a risk factor for early liver graft failure, and it is recommended that it be corrected, at least to less than $155 \mathrm{mEq} / \mathrm{L}$, prior to the organ recovery [2].

While studies have shown that maintaining a lower CVP does not have a detrimental effect on kidney outcomes $[14,23]$, when the lungs are not being considered, more aggressive fluid management may be warranted, as most guidelines still suggest that renal viability may be at risk in absence of liberal hydration [2].

\section{COVID-19 considerations}

As the world is currently in the midst of a global pandemic, there has been an impact on all aspects of life as we know it, including organ donation. The number of patients in need of life saving transplants has not diminished, so it is imperative to continue to do everything possible to ensure the availability of this life-saving gift of the lungs. It is also vital to ensure that the gifts provided are being done so safely. This includes testing all donors for severe acute respiratory syndrome coronavirus 2 (SARS-CoV-2). In addition, due to the risk of an infection in the lungs being present even with a negative upper respiratory (nasopharyngeal swab) test, per the OPTN it is required that all potential deceased lung donors have nucleic acid testing (NAT) for coronavirus disease 2019 (COVID-19) by lower respiratory specimen, obtained via a tracheal aspirate or bronchoalveolar lavage, to ensure recipient safety.

\section{Conclusion}

Optimal donor organ management is extremely important for transplant outcomes. As long as the number of available organ donors remains insufficient to prevent the deaths of those patients who are on the transplant waiting lists, the best practices for management of the organ donor and maximizing donor yield must continue to be discussed.

Funding None.

\section{Declarations}

Ethics approval Not applicable as per institutional ethical committee as the scientific information presented in paper does not fall into category of clinicals trial or usage of experimental modalities.

Informed consent Not applicable.

Conflict of interest The author declares no competing interests.

\section{References}

1. Gift of Life Donor Program. www.donors1.org. Accessed 30 Jan 2021.

2. Kotloff RM, Blosser S, Fulda GJ, et al. Management of the potential organ donor in the ICU: society of critical care medicine/American college of chest physicians/association of organ procurement organizations consensus statement. Crit Care Med. $2015 ; 43: 1291-325$. 
3. Goila AK, Pawar M. The diagnosis of brain death. Indian J Crit Care Med. 2009;13:7-11.

4. Dare AJ, Bartlett AS, Fraser JF. Critical care of the potential organ donor. Curr Neurol Neurosci Rep. 2012;12:456-65. https://doi. org/10.1007/s11910-012-0272-9.

5. Maciel CB, Greer DM. ICU management of the potential organ donor: state of the art. Curr Neurol Neurosci Rep. 2016;16:86. https://doi.org/10.1007/s11910-016-0682-1.

6. Snell GI, Westall GP. Selection and management of the lung donor. Clin Chest Med. 2011;32:223-32. https://doi.org/10. 1016/j.ccm.2011.02.002.

7. Patel MS, Abt PL. Current practices in deceased organ donor management. Curr Opin Organ Transplant. 2019;24:343-50.

8. Patel MS, Niemann CU, Sally MB, et al. The impact of Hydroxyethyl starch use in deceased organ donors on the development of delayed graft function in kidney transplant recipients: a propensity-adjusted analysis. Am J Transplant. 2015;15:2152-8. https:// doi.org/10.1111/ajt.13263.

9. Bugge JF. Brain death and its implications for management of the potential organ donor. Acta Anaesthesiol Scand. 2009;53:123950. https://doi.org/10.1111/j.1399-6576.2009.02064.x.

10. Schnuelle P, Gottmann U, Hoeger S, et al. Effects of donor pretreatment with dopamine on graft function after kidney transplantation: a randomized controlled trial. JAMA. 2009;302:1067-75. https://doi.org/10.1001/jama.2009.1310.

11. Benck U, Hoeger S, Brinkkoetter PT, et al. Effects of donor pretreatment with dopamine on survival after heart transplantation: a cohort study of heart transplant recipients nested in a randomized controlled multicenter trial. J Am Coll Cardiol. 2011;58:1768-77. https://doi.org/10.1016/j.jacc.2011.05.060.

12. Novitzky D, Mi Z, Sun Q, Collins JF, Cooper DKC. Thyroid hormone therapy in the management of 63,593 braindead organ donors: a retrospective analysis. Transplantation. 2014;98:1119-27.

13. Powner DJ, Hernandez M. A review of thyroid hormone administration during adult donor care. Prog Transplant. 2005;15:202-7.

14. Abdelnour T, Rieke S. Relationship of hormonal resuscitation therapy and central venous pressure on increasing organs for transplant. J Heart Lung Transplant. 2009;28:480-5. https://doi. org/10.1016/j.healun.2009.01.018.

15. Dhar R, Stahlschmidt E, Marklin G. A randomized trial of intravenous thyroxine for brain-dead organ donors with impaired cardiac function. Prog Transplant. 2020;30:48-55. https://doi.org/10. $1177 / 1526924819893295$.
16. Peled Y, Ram E, Klempfner R, Lavee J, Cherikh WS, Stehlik J. Donor thyroid hormone therapy and heart transplantation outcomes: ISHLT transplant registry analysis. J Heart Lung Transplant. 2020;39:1070-8. https://doi.org/10.1016/j.healun.2020.06. 005.

17. Organ Procurement and Transplantation Network. https://optn. transplant.hrsa.gov/. Accessed 30 Jan 2021.

18. Miñambres E, Pérez-Villares JM, Chico-Fernández M, et al. Lung donor treatment protocol in brain dead-donors: a multicenter study. J Heart Lung Transplant. 2015;34:773-80. https://doi.org/ 10.1016/j.healun.2014.09.024.

19. Miñambres E, Pérez-Villares JM, Terceros-Almanza L, et al. An intensive lung donor treatment protocol does not have negative influence on other grafts: a multicentre study. Eur J Cardiothorac Surg. 2016;49:1719-24. https://doi.org/10.1093/ejcts/ezv454.

20. Miñambres E, Coll E, Duerto J, et al. Effect of an intensive lung donor-management protocol on lung transplantation outcomes. J Heart Lung Transplant. 2014;33:178-84. https://doi.org/10. 1016/j.healun.2013.10.034.

21. Mascia L, Pasero D, Slutsky AS, et al. Effect of a lung protective strategy for organ donors on eligibility and availability of lungs for transplantation: a randomized controlled trail. JAMA. 2010;304:2620-7. https://doi.org/10.1001/jama.2010.1796.

22. Munshi L, Keshavjee S, Cypel M. Donor management and lung preservation for lung transplantation. Lancet Respir Med. 2013;1:318-28. https://doi.org/10.1016/S2213-2600(12)70064-4.

23. Minambres E, Rodrigo E, Ballesteros MA, et al. Impact of restrictive fluid balance focused to increase lung procurement on renal function after kidney transplantation. Nephrol Dial Transplant. 2010;25:2352-6. https://doi.org/10.1093/ndt/gfq054.

24. Van Raemdonck D, Neyrinck A, Verleden GM, et al. Lung donor selection and management. Proc Am Thorac Soc. 2009;6:28-38. https://doi.org/10.1513/pats.200808-098GO.

25. Reeb J, Keshavjee S, Cypel M. Expanding the lung donor pool: advancements and emerging pathways. Curr Opin Organ Transplant. 2015;20:498-505.

Publisher's note Springer Nature remains neutral with regard to jurisdictional claims in published maps and institutional affiliations. 